\title{
MODELAGEM DE COMBUSTÍVEIS FLORESTAIS NO PARQUE NACIONAL DO IGUAÇU, PR, BRASIL
}

\author{
Luiz Antonio Mota Nunes de Melo*, Ronaldo Viana Soares**, Joésio Deoclécio Pierin Siqueira***, \\ Flávio Felipe Kirchner*** \\ *Eng. Florestal, M.Sc., IBAMA - nunesdemelo@uol.com.br \\ **Eng. Florestal, Ph.D., Depto. de Ciências Florestais, UFPR - rvsoares@ufpr.br \\ ***Eng. Florestal, Dr., Depto. de Ciências Florestais, UFPR - joesio@matrix.com.br; kirchner@floresta.ufpr.br
}

Recebido para publicação: 03/04/2006 - Aceito para publicação: 10/07/2006

\begin{abstract}
Resumo
O objetivo principal deste trabalho foi o desenvolvimento de equações matemáticas que estimem a carga de combustível superficial existente nas florestas do Parque Nacional do Iguaçu. O conhecimento da quantidade de combustível existente é importante para a previsão do comportamento do fogo caso ocorra um incêndio florestal na área. Os combustíveis coletados, vivos e mortos, em 133 parcelas de 1,0 x 1,0 m, foram classificados de acordo com suas naturezas e seus diâmetros, originando os seguintes grupos: miscelânea (MIS); combustíveis mortos com diâmetro $\leq 0,7 \mathrm{~cm}$ (L1M); combustíveis mortos com diâmetro $>0,7 \mathrm{~cm}$ (L2M); combustíveis lenhosos mortos (LTM = L1M + L2M; total de combustíveis mortos (MLTM = MIS + LTM); combustíveis vivos com diâmetro $\leq 0,7$ cm (L1V); combustíveis vivos com diâmetro > 0,7 cm (L2V); total de combustíveis vivos (LTV = L1V + L2V); e carga total de combustíveis $(\mathrm{CTC}=\mathrm{MLTM}+\mathrm{LTV})$. As variáveis independentes medidas e usadas na modelagem foram: espessura da manta orgânica (EMO); diâmetro médio à altura do peito (DAP); área basal (G); altura média das árvores (H). As cargas totais de combustíveis obtidas variaram de 1,75 a 21,72 T. ha ${ }^{-1}$, com uma média de 11,74 T. $\mathrm{ha}^{-1}$. O melhor modelo para estimar a carga de combustíveis mortos foi $\left(\mathrm{R}^{2}=0,57\right)$ : MLTM $=951,639+$ 20,179 $\left(\mathrm{EMO}^{3}\right)-800,441(1 / \mathrm{EMO})-0,090\left(\mathrm{G}^{2 *} \mathrm{EMO}\right)+14,982(\mathrm{G})$. O melhor modelo para estimar a carga total de combustível foi $\left(\mathrm{R}^{2}=0,58\right): \mathrm{CTC}=1162,193+19,355\left(\mathrm{EMO}^{3}\right)-925,90(1 / \mathrm{EMO})-0,086$ $\left(\mathrm{G}^{2 *} \mathrm{EMO}\right)+13,980(\mathrm{G})$. Os valores obtidos na amostragem não permitiram o desenvolvimento de um modelo válido para estimar a carga de combustíveis vivos, devido aos baixos coeficientes de determinação. Palavras chave: Incêndios florestais; biomassa; serapilheira; Parque Nacional do Iguaçu; proteção florestal.
\end{abstract}

\section{Abstract}

Forest fuel load modeling in the Iguaçu National Park, PR - Brazil. The objectives of this research were to measure and model the surface forest fuel in the Iguaçu National Park. A total of 133 plots $(1.0 \mathrm{mx} 1.0 \mathrm{~m})$ were located in the Park area in order to collect the information needed to develop the investigation. Live and dead woody surface fuels were collected, separated and classified, generating the following groups: miscellanea (MIS); dead fuel, diameter $\leq 0.7 \mathrm{~cm}(\mathrm{~L} 1 \mathrm{M})$; dead fuel, diameter $>0.7 \mathrm{~cm}$ (L2M); total woody dead fuel $(\mathrm{LTM}=\mathrm{L} 1 \mathrm{M}+\mathrm{L} 2 \mathrm{M})$; total dead fuel (MLTM = MIS + LTM); live fuel, diameter $\leq 0.7 \mathrm{~cm}$ (L1V); live fuel, diameter $>0.7 \mathrm{~cm}(\mathrm{~L} 2 \mathrm{~V})$; total woody live fuel (LTV $=\mathrm{L} 1 \mathrm{~V}+\mathrm{L} 2 \mathrm{~V})$; and total fuel load $(\mathrm{CTC}=\mathrm{MLTM}+\mathrm{LTV})$. The independent variables measured were: litter depth (EMO); average DBH (DAP); basal area $(\mathrm{G})$; and trees average height $(\mathrm{H})$. The total fuel load measured through the sample plots varied from 1.75 to 21.72 ton.ha ${ }^{-1}$, with an average of 11.74 ton.ha ${ }^{-1}$. Models generated through the forward stepwise methodology could be considered a first approach to estimate the fuel load based on some easily obtained independent variables in the Iguaçu National Park. The best model to estimate total dead fuel load was $\left(\mathrm{R}^{2}=0.57\right):$ MLTM $=951.639+20.179\left(\mathrm{EMO}^{3}\right)-800.441(1 / \mathrm{EMO})-0,090\left(\mathrm{G}^{2} * \mathrm{EMO}\right)+14.982(\mathrm{G})$ and the best model to estimate the total fuel load was $\left(\mathrm{R}^{2}=0.58\right)$ : CTC $=1162.193+19.355\left(\mathrm{EMO}^{3}\right)-$ $925.90(1 / \mathrm{EMO})-0.086\left(\mathrm{G}^{2} * \mathrm{EMO}\right)+13.980(\mathrm{G})$. It was not possible to generate a model to estimate live fuel load due to the lower determination coefficients obtained.

Keywords: Forest fires; forest fuel; biomass; Iguaçu National Park.

\section{INTRODUÇÃO}

Os incêndios constituem-se numa das maiores ameaça aos reflorestamentos e florestas nativas do planeta. Por isso é necessária a implantação de eficientes sistemas integrados de proteção contra incêndios florestais tanto em florestas produtivas como em Unidades de Conservação. 
Essa necessidade é ressaltada pela dificuldade econômica e física para manter baixos os níveis de combustíveis acumulados em áreas de florestas, nativas ou reflorestadas, de forma a minimizar o risco de propagação de um incêndio.

As previsões dos incêndios florestais não podem estar somente ligadas às condições meteorológicas do momento. Para entender o comportamento de um incêndio florestal, é necessário ter o pleno conhecimento das particularidades dos combustíveis presentes. Não há como se fazer estimativas do comportamento dos incêndios florestais sem considerar as características físicas e químicas dos combustíveis que irão determinar, desde a ignição, o seu comportamento posterior e a energia liberada até a dificuldade de controle e extinção desses incêndios.

Embora um inventário seja a forma mais precisa de quantificação do combustível florestal, o tempo despendido na sua realização é muito longo, o que inviabiliza sua utilização nos casos em que o incêndio já iniciou e se pretende estimar a intensidade do fogo. Nessas situações, o mais indicado é recorrer aos modelos de combustível para avaliar as características do material combustível existente numa floresta.

Os modelos permitem, através de variáveis independentes, como área basal e espessura da manta orgânica, estimar as cargas dos combustíveis florestais, assim como seus componentes, principalmente serapilheira e material lenhoso vivo e morto.

A modelagem de combustíveis no Parque Nacional do Iguaçu representa uma grande contribuição para a estimativa da intensidade de eventuais incêndios bem como para o planejamento do combate ao fogo, incluindo os meios materiais necessários para contê-lo e evitar danos à vegetação e demais recursos e equipamentos do Parque.

Os objetivos principais deste trabalho foram:

- Determinar a carga dos combustíveis florestais das diferentes tipologias do Parque Nacional do Iguaçu.

- Desenvolver equações matemáticas, baseadas em variáveis de fácil obtenção, para se estimar a carga dos combustíveis florestais no Parque Nacional do Iguaçu, contribuindo assim para o conhecimento do comportamento do fogo em eventuais incêndios que venham a ocorrer nas suas florestas nativas.

\section{MATERIAL E MÉTODOS}

\section{Caracterização da área de estudo}

\section{Localização}

Este trabalho foi desenvolvido no Parque Nacional do Iguaçu (PNI), Unidade de Conservação do tipo proteção integral, instituído através do Decreto Federal n 1.035, de 10 de janeiro de 1939.

A Lei $\mathrm{n}^{\circ} 9.985$ de 18 de julho de 2.000, instituiu o Sistema Nacional de Unidades de Conservação (SNUC), estabelecendo critérios e normas para a criação, implantação e gestão das Unidades de Conservação, com o objetivo principal de proteger a flora, a fauna e as belezas cênicas das Cataratas do Iguaçu. Considerado a maior Unidade de Conservação de Proteção Integral da Região Sul do Brasil, o Parque Nacional do Iguaçu está localizado no estado do Paraná (Figura 1), incluindo partes dos municípios de Céu Azul (49,56\%), Foz do Iguaçu (7,48\%), Matelândia (19,87\%), São Miguel do Iguaçu $(11,73 \%)$ e Serranópolis do Iguaçu (16,92\%). Margeiam ainda o Parque os municípios de Capanema, Capitão Leônidas Marques, Lindoeste, Santa Lúcia, Santa Tereza do Oeste e Santa Terezinha de Itaipu. Com área estimada em 185.265,50 ha, o Parque Nacional do Iguaçu apresenta um perímetro aproximado de $420 \mathrm{~km}$, sendo $300 \mathrm{~km}$ constituídos por limites naturais. Seu gigantesco banco genético verde de valor incalculável e a exuberância dos cenários naturais fizeram-no ser considerado, pela UNESCO, como Patrimônio Natural da Humanidade.

\section{Clima}

O clima da região, segundo a classificação de Koeppen, é do tipo Cfa, subtropical úmido ou mesotérmico com verão quente, apresentando pluviosidade acima de $900 \mathrm{~mm}$, igualmente distribuída ao longo do ano e temperaturas médias entre $15{ }^{\circ} \mathrm{C}$ e $25{ }^{\circ} \mathrm{C}$. De maneira geral, o regime de chuvas reflete o comportamento de clima de transição, apresentando índices pluviométricos elevados, entre $1500 \mathrm{~mm}$ a $2000 \mathrm{~mm}$ anuais. Os meses de outubro, novembro, dezembro e janeiro são os mais chuvosos, sendo que o 
mês de novembro é o que apresenta historicamente a menor pluviosidade. A intensidade média dos ventos é em torno de $11 \mathrm{~km} / \mathrm{h}$, não apresentando variações significativas ao longo do ano.

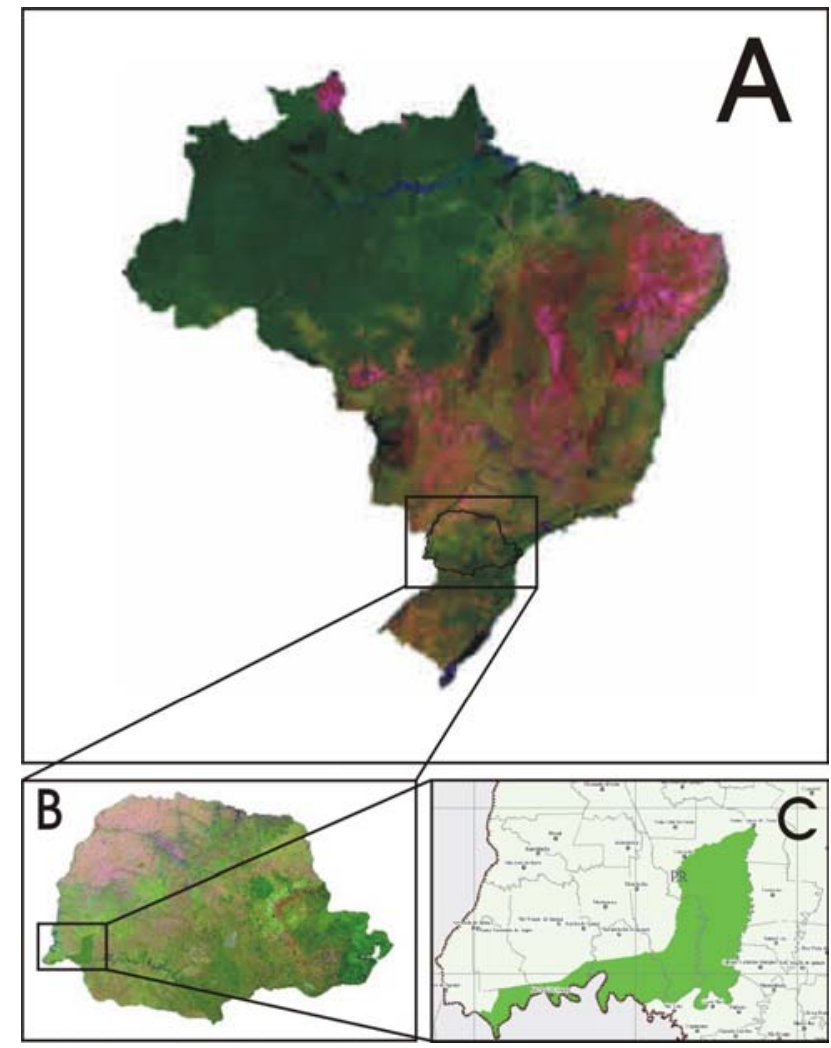

Figura 1. Área de estudo: A - mapa do Brasil com o Paraná em destaque; B - mapa do Paraná com a área do Parque Nacional do Iguaçu em destaque (C).

Figure 1. Study area: A - Brazil map with Parana State in detail; B - Parana State map with Iguaçu National Park (C) in detail.

Vegetação

O Parque Nacional do Iguaçu é uma Unidade de Conservação de proteção integral onde estão inseridos dois biomas distintos:

- Floresta Ombrófila Mista: conhecida como "Floresta com araucária", constitui uma das mais importantes formações florestais do Sul do Brasil, não só pela área que outrora ocupava, mas também pelo papel que os seus recursos naturais representaram para a região. Sua área central de ocorrência está praticamente restrita ao Planalto Meridional dos estados do Paraná, Santa Catarina e Rio Grande do Sul, sendo que outrora essa formação ainda ocorria, na forma de "ilhas", ao longo das partes mais altas da Serra da Mantiqueira, até praticamente a divisa do Rio de Janeiro com o Espírito Santo. Distribui-se ainda em países vizinhos ao Brasil, notadamente no nordeste da Argentina e sudeste do Paraguai, neste último em área pouco expressiva (LEITE; KLEIN, 1990; VELOSO et al., 1991).

- Floresta Estacional Semidecidual: esse tipo florestal caracteriza-se por comunidades onde 20 a 50\% dos indivíduos do estrato arbóreo superior perdem as folhas na estação desfavorável e, portanto, está relacionada em praticamente toda a sua área de ocorrência a um clima de duas estações definidas, uma chuvosa e outra seca (Nordeste, Centro-Oeste e parte do Sudeste), ou então a uma acentuada variação térmica (Sul). Sendo assim, ela ocorre de maneira descontínua praticamente em todos os estados das regiões Nordeste, Sudeste e Sul do país, e em parte da Centro-Oeste, chegando até a bacia do rio Uruguai, ao Paraguai e à Argentina (VELOSO et al., 1991). 


\section{Amostragem/análise estatística}

Devido à diversidade de formações florestais e às ações antrópicas anteriores à criação da Unidade de Conservação, não houve condições de estratificação da vegetação. Mesmo com o apoio das cartas com base aerofotogramétrica de 1952/1953, as áreas anteriormente antropizadas, hoje florestas secundárias que se confundem com as primárias, não puderam ser diferenciadas.

A coleta do material combustível foi realizada entre os meses de julho e agosto de 2004 . As unidades da amostra, proporcionalmente distribuídas em cada uma das tipologias, foram localizadas inteiramente ao acaso, ou seja, as $N$ unidades da população tiveram igual possibilidade de participar do processo de amostragem. No total foram coletadas 133 amostras, como mostra a figura 2. O número mínimo de amostras foi estimado pelo método seqüencial de Stein (STEEL; TORRIE, 1960).

Para a coleta dos dados, foram utilizadas parcelas 1,0 $\mathrm{m}$ x 1,0 m, de acordo com os trabalhos de Soares (1979), Batista (1984 e 1995), Schneider e Bell (1985), Ribeiro (1997) e Souza (2000).

As variáveis dependentes foram os pesos dos materiais combustíveis mortos, materiais combustíveis vivos e carga total do material combustível. Devido à pequena participação de alguns combustíveis coletados nas unidades amostrais, que poderiam ser insignificantes sob o ponto de vista estatístico, eles foram incluídos em classes semelhantes, resultando a seguinte composição:

- $\quad$ Miscelânea $\Rightarrow$ MIS

- $\quad$ Combustíveis mortos com diâmetro $\leq 0,7 \mathrm{~cm} \Rightarrow \mathrm{L} 1 \mathrm{M}$

- Combustíveis mortos com diâmetro $>0,7 \mathrm{~cm} \Rightarrow \mathrm{L} 2 \mathrm{M}$

- Combustíveis mortos, somatória de L1M + L2M $\Rightarrow$ LTM

- Combustíveis mortos total, somatória de MIS + LTM $\Rightarrow$ MLTM

- Combustíveis vivos com diâmetro $\leq 0,7 \mathrm{~cm} \Rightarrow \mathrm{L} 1 \mathrm{~V}$

- Combustíveis vivos com diâmetro $>0,7 \mathrm{~cm} \Rightarrow \mathrm{L} 2 \mathrm{~V}$

- Combustíveis vivos, somatória de L1V + L2V $\Rightarrow$ LTV

- $\quad$ Carga total, somatória de MIS + LTM + LTV $\Rightarrow$ CTC

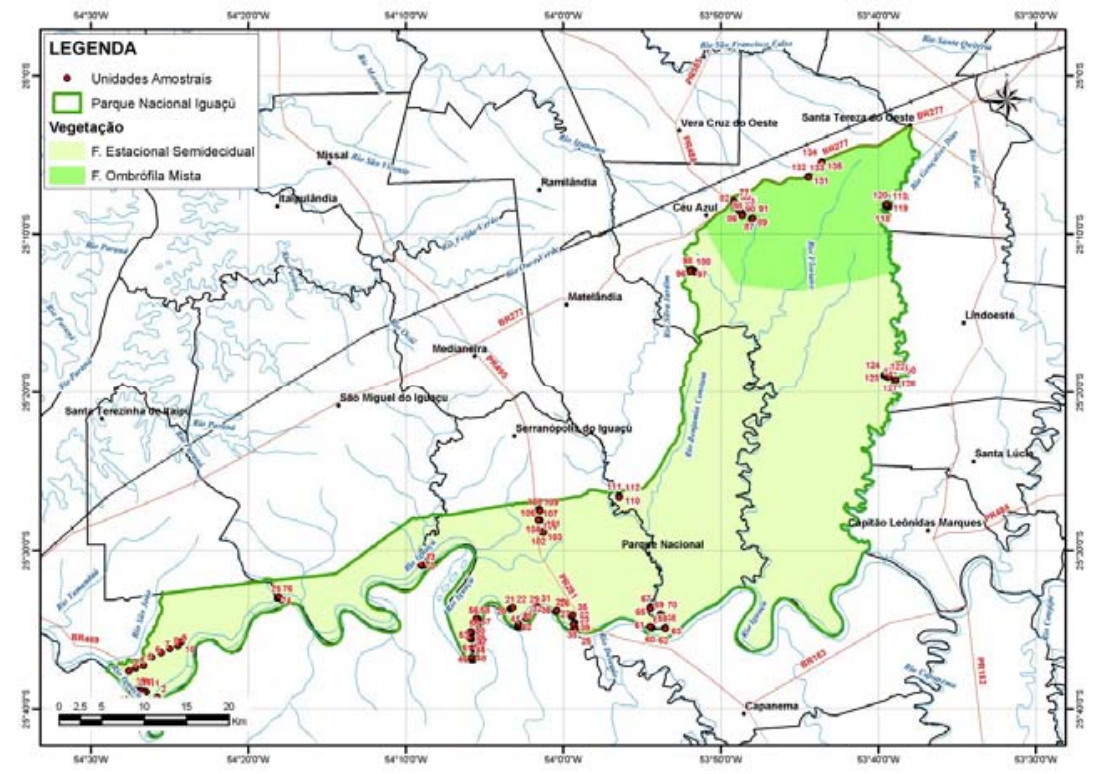

Figura 2. Localização das unidades amostrais no interior do Parque Nacional do Iguaçu.

Figure 2. Sample plots location inside the Iguaçu National Park.

As variáveis independentes definidas e medidas para se iniciar a modelagem dos combustíveis foram: espessura da manta orgânica $(\mathrm{EMO})$, área basal $(\mathrm{G})$, altura média $(\mathrm{H})$ e diâmetro à altura do peito (DAP) das árvores em torno das parcelas de $1,0 \mathrm{~m}^{2}$. Para a medição das três últimas variáveis, foram 
instaladas parcelas de 10,0 m x 10,0 m, sendo um dos seus vértices comum a um dos vértices da unidade amostral de 1,0 m x 1,0 m de coleta de combustíveis. A cada três parcelas de 1,0 $\mathrm{m}^{2}$ medidas, era lançada uma parcela de $10,0 \mathrm{~m} \times 10,0 \mathrm{~m}$, no interior das quais foram medidas a altura e o DAP médios.

\section{Coleta dos combustíveis}

A delimitação das parcelas foi feita com o auxílio de um gabarito de alumínio (Figura 3), dobrados em segmentos de $1,0 \mathrm{~m}$, fechado com parafuso de rosca, com um calibrador de $90^{\circ}$ para formar um quadrado. As coordenadas das parcelas foram definidas com o uso de um GPS.

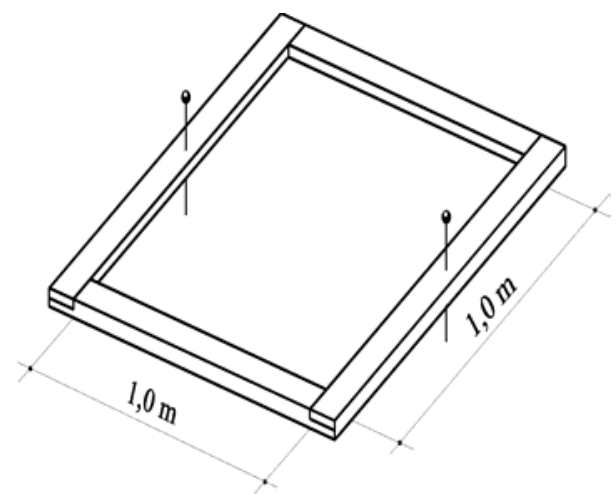

Figura 3. Gabarito usado para delimitar as parcelas.

Figure 3. Gauge used to limit the plots areas.

Inicialmente, com um serrote e uma tesoura de poda, foi retirada toda a vegetação viva até $1,80 \mathrm{~m}$ de altura, limite do combustível superficial. Esse material foi classificado por seu diâmetro (BROWN, 1974), com o auxílio de um calibrador (Figura 4), em lenhoso 1 (diâmetro $\leq 0,7 \mathrm{~cm}$ ), lenhoso $2(0,71$ a 2,5 cm), lenhoso $3(2,5$ a 7,5 cm) e lenhoso 4 (diâmetro $>7,5 \mathrm{~cm}$ ). A pesagem do material classificado foi feito através de balança de $2,610 \mathrm{~kg}$ de capacidade, com precisão de $0,1 \mathrm{~g}$.

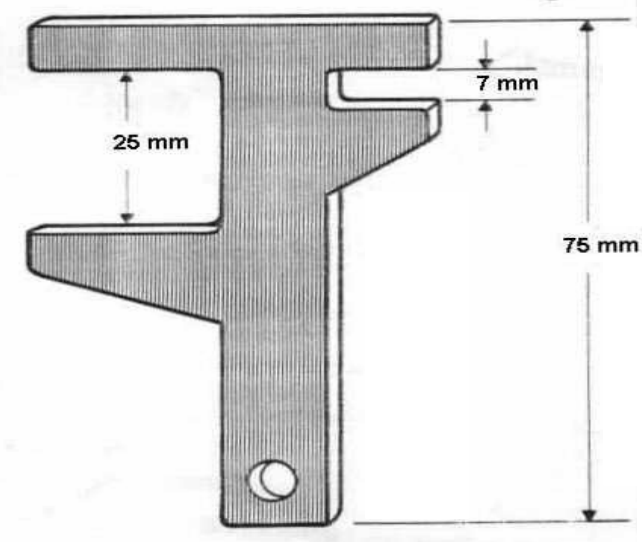

Figura 4. Calibrador usado na classificação dos combustíveis.

Figure 4. Gauge used to classify the woody fuels.

Com uma régua graduada com precisão de $0,1 \mathrm{~cm}$, foram medidas, em cada vértice do quadrado, as espessuras da manta orgânica, obtendo-se assim a espessura média de cada parcela, que foi uma das variáveis independentes usadas na análise estatística.

Depois de coletado o combustível vivo e medida a espessura da manta orgânica, iniciou-se o processo de coleta e classificação do material combustível morto. Primeiramente, o material lenhoso foi 
separado e classificado da mesma maneira que o material vivo. Posteriormente foi coletada, com auxílio de um mini-rastelo, a miscelânea (folhas, estróbilos, gramíneas secas e materiais semidecompostos).

Após a operação de coleta, classificação e pesagem, foram retiradas amostras compostas de cada classe que, após pesadas, foram colocadas em sacos plásticos individuais, devidamente etiquetados, para serem levados ao laboratório para secagem e posterior determinação do teor de umidade e peso da matéria seca ao forno. A secagem foi feita no laboratório do Parque Nacional do Iguaçu, a uma temperatura de \pm $75^{\circ} \mathrm{C}$, até o material atingir peso constante, o que foi obtido após aproximadamente 48 horas.

A organização dos dados para a obtenção dos modelos para estimativa de biomassa teve seu início com a elaboração de uma planilha onde foram separadas as variáveis dependentes, que são todos os componentes dos combustíveis florestais vivos e mortos medidos e pesados (MIS, L1M, L2M, LTM, L1V, L2V, LTV, TOTAL), e as independentes, circunferência à altura do peito, diâmetro à altura do peito, área basal, altura total, espessura da manta e área basal.

Como havia poucos modelos testados na literatura para a estimativa da biomassa em florestas nativas, foram desenvolvidos novos modelos a partir da escolha de variáveis pelo método "forward stepwise", utilizando o programa "Statistica for Windows" 6.0, em computador do laboratório de Incêndios Florestais do DECIF/UFPR. O modelo máximo utilizou as seguintes variáveis independentes:

- Espessura da manta orgânica $(\mathrm{mm}) \Rightarrow(\mathrm{EMO})$

- Área basal $\left(\mathrm{m}^{2} \cdot \mathrm{ha}^{-1}\right) \Rightarrow(\mathrm{G})$

- $\quad$ Altura $(\mathrm{m}) \Rightarrow(\mathrm{H})$

- $\operatorname{CAP}(\mathrm{m})$

- $\operatorname{DAP}(\mathrm{m})$

- $\mathrm{DAP}^{2} \cdot \mathrm{H}$

- DAP x Espessura da manta orgânica $\Rightarrow$ (DAP.EMO)

- $\mathrm{G}^{2} \times$ Espessura da manta orgânica $\Rightarrow\left(\mathrm{G}^{2} . \mathrm{EMO}\right)$

- Espessura da manta orgânica $\Rightarrow\left(\mathrm{EMO}^{3}\right)$

- $\mathrm{G}^{2} \cdot \mathrm{H}$

- $\quad 1$ / Espessura da manta orgânica $\Rightarrow(1 /$ EMO $)$

- $\ln \left(\mathrm{DAP}^{2} \cdot \mathrm{H}\right)$

- $\quad \ln$ (Espessura da manta orgânica) $\Rightarrow \ln ($ EMO)

O procedimento de seleção "forward" parte da variável independente, mas correlacionada com a variável dependente para, no passo seguinte, incluir mais uma variável que apresentar maior correlação parcial e posteriormente serem testadas pelo teste F (SCHNEIDER, 1998). O método pára de incluir variáveis no ponto em que a adição de mais uma variável mostrar um $\mathrm{F}$ calculado não significativo.

\section{RESULTADOS E DISCUSSÃO}

\section{Quantificação da carga de combustível}

Para a determinação da carga de combustível, foram medidas 133 (cento e trinta e três) amostras, das quais 3 (três) foram descartadas por apresentarem resultados inconsistentes. $\mathrm{O}$ número de amostras, calculado através do método seqüencial de Stein (STEEL; TORRIE, 1960), com 10\% de erro, mostrou que as 130 (cento e trinta) amostras foram suficientes para o desenvolvimento do trabalho.

As cargas totais de combustíveis encontradas nas duas tipologias foram semelhantes (Tabela 1), apresentando 11,51 ton.ha ${ }^{-1}$ na floresta ombrófila mista e 11,96 ton.ha ${ }^{-1}$ na floresta estacional semidecidual. Caldeira (2003), trabalhando em uma Floresta Ombrófila Mista em General Carneiro (PR), encontrou valores médios de serapilheira variando de 4,43 a 13,71 ton.ha ${ }^{-1}$.

\section{Correlações entre as variáveis independentes e dependentes}

Os coeficientes de correlação entre as variáveis independentes (espessura da manta orgânica, altura média das árvores, diâmetro médio das árvores e área basal) e dependentes (lenhoso vivo 1, lenhoso vivo 2, lenhoso vivo total, lenhoso morto 1 , lenhoso morto 2 , lenhoso morto total, morto total, miscelânea e total) podem ser observados na tabela 2. 
Tabela 1. Carga média de combustível (ton.ha ${ }^{-1}$ da matéria seca ao forno), por componente e por tipologia florestal.

Table 1. Oven dry fuel load (ton.ha ${ }^{-1}$ ), by component and forest type.

\begin{tabular}{lcc}
\hline Componente & Floresta Ombrófila Mista & Floresta Estacional Semidecidual \\
\hline L1V (vivo $\varnothing \leq 0,7)$ & 0,613201 & 0,651645 \\
L2V (vivo $\varnothing>0,7)$ & 0,051179 & 0,434801 \\
LTV (vivo total) & 0,66438 & 1,086447 \\
L1M (morto $\varnothing \leq 0,7)$ & 0,74898 & 0,47528 \\
L2M (morto $\varnothing>0,7)$ & 1,223012 & 1,410077 \\
LTM (morto lenhoso) & 1,971992 & 1,885357 \\
MIS (miscelânea) & 9,327476 & 8,536874 \\
MT (morto total) & 11,299468 & 10,422231 \\
Total & 11,50868 & 11,96385 \\
\hline
\end{tabular}

Tabela 2. Coeficientes de correlação entre variáveis medidas e as classes de material combustível no PNI.

Table 2. Correlation coefficients between the measured variables and the fuel classes.

\begin{tabular}{|c|c|c|c|c|}
\hline \multirow{2}{*}{$\begin{array}{l}\text { Variáveis } \\
\text { dependentes }\end{array}$} & \multicolumn{4}{|c|}{ Variáveis independentes } \\
\hline & $\begin{array}{c}\text { Espessura manta } \\
\text { orgânica }\end{array}$ & $\begin{array}{c}\text { Altura média das } \\
\text { árvores }\end{array}$ & $\begin{array}{l}\text { DAP médio das } \\
\text { árvores }\end{array}$ & Área basal \\
\hline L1V & $0,2111^{*}$ & 0,0886 & 0,1371 & $-0,0202$ \\
\hline L2V & 0,0315 & $-0,0714$ & $-0,1869$ & $-0,0777$ \\
\hline LTV & 0,1039 & $-0,0610$ & $-0,1189$ & $-0,0771$ \\
\hline L1M & $0,3312 *$ & 0,1264 & 0,1606 & 0,1157 \\
\hline L2M & $0,3562 *$ & $-0,0404$ & $-0,0016$ & 0,0741 \\
\hline LTM & $0,4051 *$ & $-0,0123$ & 0,0315 & 0,0939 \\
\hline MLTM & $0,6675^{*}$ & $-0,0325$ & 0,0754 & 0,1603 \\
\hline MIS & $0,6487 *$ & $-0,0342$ & 0,0782 & 0,1570 \\
\hline Total & $0,6855^{*}$ & $-0,0436$ & 0,0535 & 0,1460 \\
\hline
\end{tabular}

* Significativas ao nível de $90 \%$.

A espessura da manta orgânica foi a única variável independente que apresentou correlações significativas com alguns componentes dos combustíveis, principalmente com a miscelânea, o lenhoso total morto e o total morto. Nas florestas nativas, a variabilidade das características florestais, como espécies vegetais, presença de lianas e cipós, diâmetro de copas, alturas e diâmetros de tamanhos variados, e ainda a queda extemporânea de folhas e a presença de sub-bosque, faz com que haja grande diferença entre as florestas nativas e as plantadas. Os valores das variáveis independentes observados na amostragem estão apresentados na tabela 3 . Na figura 4 é mostrada a relação entre a espessura da manta orgânica e a carga total média de material combustível.

Tabela 3. Valores obtidos para as variáveis independentes.

Table 3. Independent variables values measured in the field.

\begin{tabular}{lccc}
\hline Variável independente & Valor mínimo & Valor médio & Valor máximo \\
\hline Espessura da manta $(\mathrm{cm})$ & 1,0 & 2,0 & 5,0 \\
Altura média das árvores $(\mathrm{m})$ & 4,2189 & 7,5909 & 11,2500 \\
DAP médio $(\mathrm{cm})$ & 5,0285 & 10,6036 & 17,3567 \\
Área basal $\left(\mathrm{m}^{2} \cdot \mathrm{ha}^{-1}\right)$ & 6,6300 & 30,0700 & 92,8700 \\
\hline
\end{tabular}

Cole; Rapp (1980) observaram em suas pesquisas que as florestas de coníferas produzem menos serapilheira do que florestas latifoliadas. Da mesma forma, neste trabalho, a floresta de conífera 
(ombrófila mista) produziu menos combustível que a floresta latifoliada (estacional semidecidual), embora a diferença tenha sido muito pequena.

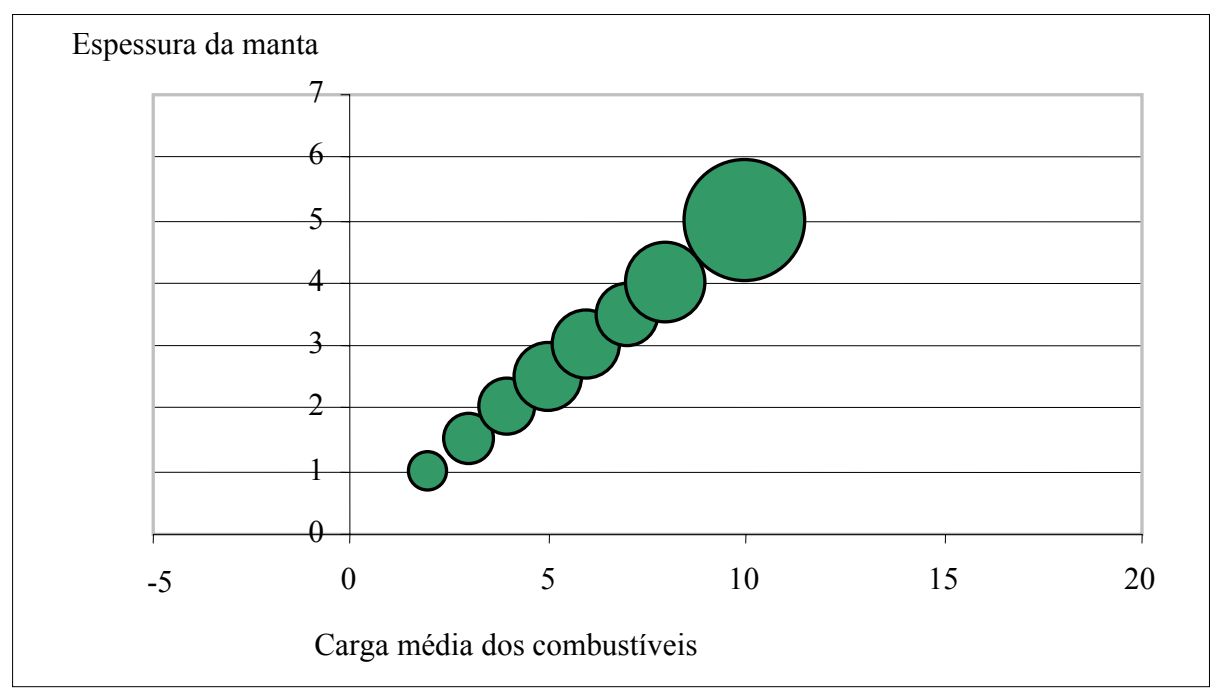

Figura 4. Relação entre espessura da manta orgânica $(\mathrm{cm})$ e a carga total de combustíveis (ton.ha $\left.{ }^{-1}\right)$. Figure 4. Relationship between the litter layer depth $(\mathrm{cm})$ and the total fuel load (ton.ha ${ }^{-1}$ ).

\section{Modelagem dos combustíveis}

A escolha da melhor equação para estimar as diversas classes de combustível foi baseada no coeficiente de determinação ou no número de variáveis independentes, quando duas ou mais equações apresentavam $\mathrm{R}^{2}$ semelhantes. Isso significa que foram selecionadas as equações com maior $\mathrm{R}^{2}$ e/ou menor número de variáveis independentes.

\section{Miscelânea (MIS)}

A melhor equação para estimar a carga da miscelânea incluiu as variáveis independentes espessura da manta orgânica, área basal, diâmetro médio e altura média $\left(\mathrm{R}^{2}=0,576\right)$ :

$$
\mathrm{MIS}=141,7676+16,9160\left(\mathrm{EMO}^{3}\right)-0,0778\left(\mathrm{G}^{2} . \mathrm{EMO}\right)+10,3663(\mathrm{G})+14,3586(\mathrm{DAP} . \mathrm{EMO})
$$

\section{Combustível morto total (MLTM)}

Essa classe é composta pelos combustíveis lenhosos mortos e pela miscelânea. A melhor equação para estimar a carga total de combustíveis mortos incluiu a espessura da manta orgânica e a área basal da floresta $\left(\mathrm{R}^{2}=0,572\right)$ :

$\mathrm{MLTM}=951,639+20,179\left(\mathrm{EMO}^{3}\right)-800,441(1 / \mathrm{EMO})-0,090\left(\mathrm{G}^{2} . \mathrm{EMO}\right)+14,982(\mathrm{G})$

A inclusão do DAP elevaria o $\mathrm{R}^{2}$ para 0,58 , mas o ganho em precisão não compensaria a utilização de mais uma variável independente.

\section{Carga total de combustíveis (CTC)}

Essa classe foi formada pela somatória dos combustíveis vivos e mortos. A melhor equação para estimá-la incluiu também as variáveis espessura da manta orgânica e a área basal $\left(\mathrm{R}^{2}=0,581\right)$ :

$$
\mathrm{CTC}=1162,193+19,355\left(\mathrm{EMO}^{3}\right)-925,90(1 / \mathrm{EMO})-0,086\left(\mathrm{G}^{2} . \mathrm{EMO}\right)+13,980(\mathrm{G})
$$

Os coeficientes de determinação calculados para as três equações apresentadas foram relativamente baixos. Isso se deve à grande variabilidade existente em florestas nativas. Sackett (1976), por exemplo, estimando a carga de combustível superficial em florestas de Pinus ponderosa (mais homogênea do que uma floresta latifoliada), nos estados americanos do Arizona e Novo México, utilizando como variável independente a área basal, encontrou um $\mathrm{R}^{2}=0,56$. 
Em plantações florestais, muito mais homogêneas do que as florestas nativas, é possível desenvolver modelos mais precisos. Brender; McNab; Williams (1976) desenvolveram equações para estimar o combustível superficial morto em plantações de Pinus taeda no estado da Geórgia, EUA, com coeficientes de determinação de 0,77 a 0,79 . Souza (2000) desenvolveu equações para a estimativa das cargas totais de combustíveis em plantações de Eucalyptus dunnii e Pinus taeda no estado de Santa Catarina, com $\mathrm{R}^{2}=0,68$ e $\mathrm{R}^{2}=0,70$, respectivamente.

\section{CONCLUSÕES}

Os resultados obtidos nesta pesquisa permitem as seguintes conclusões:

- A carga total média de combustível superficial medida nas florestas do Parque Nacional do Iguaçu foi de 11,899 ton.ha ${ }^{-1}$, variando de 2,108 a 52,940 ton.ha ${ }^{-1}$.

- A variabilidade encontrada no material amostrado dificultou a obtenção de modelos matemáticos com bom ajuste para melhor estimar a carga de combustíveis no PNI.

- O material superficial vivo apresentou grande variabilidade, o que impossibilitou o desenvolvimento de uma equação para estimá-lo.

- A espessura da manta orgânica foi a variável independente que melhor se correlacionou com as cargas de combustível.

- A estimativa de carga total de combustível total foi a que apresentou o modelo com melhor ajuste, utilizando como variáveis independentes a espessura da manta orgânica e a área basal.

\section{REFERÊNCIAS}

BATISTA, A. C. Avaliação da queima controlada em povoamentos de Pinus taeda L. no norte do Paraná. 108f. Tese (Doutorado em Engenharia Florestal) - Setor de Ciências Agrárias, Universidade Federal do Paraná, Curitiba, 1995.

BATISTA, A. C. Determinação de unidade do material combustível sob povoamento de Pinus taeda L. 61f. Dissertação (Mestrado em Engenharia Florestal) - Setor de Ciências Agrárias, Universidade Federal do Paraná, Curitiba, 1984.

BRAY, J. R.; GOHRAM, E. Litter production in the forests of the world. Advances in ecological research, New York, v.2, p. 01-157, 1964.

BRENDER, E. V.; McNAB; WILliAMS, S. Fuel accumulations in Piedmont loblolly pine plantations. Asheville: USDA Forest Service, 1976. 4p. (Research Note SE-233).

BROWN, A. A.; DAVIS, K. P. Forest fire: control and use. 2.ed. New York: McGraw Hill Book, 1973.

CALDEIRA, M. V. W. Determinação de biomassa e nutrientes em uma Floresta Ombrófila Mista Montana em General Carneiro, Paraná. 176f. Tese (Doutorado em Engenharia Florestal) - Setor de Ciências Agrárias, Universidade Federal do Paraná, Curitiba, 2003,

CARLTON, D. W.; PICKFORD, S. G. Fuel bed changes with aging of slash from ponderosa pine thinning. Journal of Forestry, Washington, DC, v.80, n.2, p.105-108, 1982.

CHANDLER, C.; CHENEY, P.; THOMAS, P.; TRABAUD, L.; WILLIAMS, D. Fire in forestry: Forest fire behavior and effects. New York: Wiley \& Sons, 1983.

COLE, D. W.; RAPP, M. Elemental cycling in forest ecosystems. In: REICHLE, D. E., (Ed.) Dynamic properties of forest ecosystems. Cambridge: Cambridge University Press, 1980. p.409.

DE RONDE, C.; GOLDAMMER, J. G.; WADE, D. D.; SOARES, R. V. Prescribed Fire in Industrial Pine Plantations. In: GOLDAMMER, J. G., (Ed.) Fire in the Tropical Biota. Berlin: Springer, 1990. p. 216 - 272. (ECOLOGICAL STUDIES, v.84). 
DEEMING, J. E.; LANCASTER, J. W.; FOSBERG, M. A.; FURMAN, R. W.; SCHROEDER, M. J. National Fire-Danger Rating System. Fort Collins: U.S.D.A. Forest Service,1972. (Research Paper RM, -v.84).

FULLER, M. Forest fires: An introduction to wildland fire behavior, management, firefighting, and prevention. New York: Wiley \&Sons, 1991.

GAYLOR, H. P. Wildfires: prevention and control. Bowie: Prentice-Hall, 1974.

JEFFERS, J. N. R. Modelos em ecologia. Barcelona: Oikos-tau, 1991.

KALABOKIDS, K. D.; OMI, P.N. Quadrate analysis of wildland fuel spatial variability. International Journal of Wildland Fire, Colorado, v.2, n 4, p.14 -152, 1992.

KOEHLER, C. W. Variação estacional de deposição de serapilheira e nutrientes em povoamentos de Pinus taeda na região de Ponta Grossa -PR. 148f. Tese (Doutorado em Engenharia Florestal) - Setor de Ciências Agrárias, Universidade Federal do Paraná, Curitiba, 1989.

LEITE, P. F.; KLEIN, R. M. Vegetação. In: IBGE. Geografia do Brasil; Região Sul. Rio de Janeiro, 1990. v.2.

MELLO, R. S. P. Produção de serapilheira e aspectos da ciclagem de nutrientes em dois tipos florestais adjacentes no Rio Grande do Sul. 136p. Dissertação (Mestrado em Ecologia) - Universidade Federal do Rio Grande do Sul, Porto Alegre, 1995.

MILLER, H. G. Dynamics of nutrient cycling in plantation ecosystems. In: BOWUN, G. D.; NAMBIAR, E. K. S. (Eds.) Nutrition of plantation forests. London: Academic, 1984. p.53-78.

RIBEIRO, G. A. Estudo de comportamento do fogo e de alguns efeitos da queima controlada em povoamentos de Eucalyptus viminalis Labill em Três Barras, Santa Catarina. 145f. Tese (Doutorado em Engenharia Florestal) - Setor de Ciências Agrárias, Universidade Federal do Paraná, Curitiba, 1997.

SACKETT, S. S. Forest floor fuel loadings in Pinus ponderosa in Arizona and New Mexico. [S.1.: s.n.], 1976. Trabalho não publicado, apresentado no Eastside Prescribed Fire Workshop em maio de 1976.

SACKETT, S. S.; HAASE, S. M. Fuel loading in southwestern ecosystems of the United States. North Carolina: U.S.D.A. Forest Service, 1992. p.187-192. (Research Note SE, sn).

SCHNEIDER, B. H.; BELL, D. T. A simple, effective technique for rapid measurement of fuels in low shrub communities. Australian Forest Research, Est. Melbourne, v.15, n.1, p.84, 1985.

SCHNEIDER, P. R. Análise de Regressão Aplicada - Eng ${ }^{\circ}$ Florestal. Santa Maria: UFSM. 1998. $236 \mathrm{p}$.

SOARES, R. V. Incêndios Florestais: controle e uso do fogo. Curitiba: FUPEF, 1985.

SOARES, R. V. The use of prescribed fire in Forest management in the State of Paraná, Brasil. Tese (Ph. D) - University of Washington, Seatlle, 1977.

SOUZA, L. J. B. Modelagem de material combustível em plantações de Pinus taeda L. e Eucalyptus dunnii Maidem. 127f. Tese (Doutorado em Engenharia Florestal) - Setor de Ciências Agrárias da Universidade Federal do Paraná, Curitiba, 2000.

STEEL, R. G. D.; TORRIE, J. H. Principles and procedures of statistics. New York, Mc Graw - Hill, 1960. 481 p.

VÉLEZ, R. Algunas observaciones para una selvicultura preventiva de incendios forestales. Ecología, Madrid, Fuera de Serie n.1, p.561-571, 1990.

VELOSO, H. P.; RANGE, FILHO, A. L. R.; LIMA, J. C. A. Classificação da vegetação Brasileira, adaptada a um sistema universal. Rio de Janeiro, IBGE, 1991. 123p. 